\title{
Focus of Study-Specific Interest
}

National Cancer Institute

\section{Source}

National Cancer Institute. Focus of Study-Specific Interest. NCI Thesaurus. Code C162195.

Identification of a focus of study-specific interest on or within a subject or specimen as called out in the protocol for which a measurement, test, or examination was performed. 